\title{
Phytomedical assessment of two Cymbopogon species found in Nkonkobe Municipality: toxicological effect on human Chang liver cell line
}

\author{
Beauty E. Omoruyi ${ }^{1}$ and Voster Muchenje $2^{2^{*}}$
}

\begin{abstract}
Background: Cymbopogon species are widely used as herbal remedies by the traditional healers living in Nkonkobe Municipality for the treatment and management of skin and respiratory infections. According to our survey, the plants seem to be very important because of the higher demands.

Methods: The leaves of $C$. validis and C. plurinodis were hydro-distilled and the resulted extracted oils were analyzed by GC/MS. Minimum inhibitory concentrations (MICs) ranging from 7.8 to $500.0 \mathrm{\mu g} / \mathrm{ml}$ of the extracted oils were tested against eight bacterial strains, using micro-well dilution method. The human Chang liver cell viability was determined using the CellTiter-Blue cell assay.

Results: GC-MS analysis of the C. validis essential oil amounted to $87.03 \%$, major components identified were Linalyl alcohol (18.9\%), 2-Nephthalenemethanol (6.67\%), Longifolene (6.53\%), Cubedol (6.08\%). Total oil percentage of C. plurinodis was $81.47 \%$ and the main components were characterized as 3-Cyclohexane-1-ol (13.58\%), Nerolidol (13.6\%) and 2-Carene (12.6\%). The essential oils from both plants were found to be active against the growth of Gram positive than the Gram negative bacterial tested. Lethal dose at $50\left(\mathrm{LD}_{50}\right)$ of both plants showed $74.87 \pm 1.41$ and $81.66 \pm 1.40$ degree of toxicity at $24 \mathrm{~h}$.
\end{abstract}

Conclusion: Both plants extracts were toxic to human Chang liver cell lines.

Keywords: Lemon plants, Essential oil, GC-ms, Chemical profiling, Antimicrobial activity, Toxicity evaluation

\section{Background}

There are eight known species of Cymbopogon growing in the provinces of Mpumalanga, KwaZulu-Natal, Limpopo, Gauteng, North West, Eastern Cape and Western Cape. In addition, two others namely; Cymbopogon plurinodis and $C$. validis have been identified growing abundantly in the bushveld and pasture cultivated fields around Hosback area, in Nkonkobe Municipality, in the Eastern Cape. Morphologically, each of these plant species is quite different from each other, by the presence or absence of silica thones aligned on their leaf edges, leaves bear glandular hairs, and stacked with both basal and distal cells. Both

\footnotetext{
* Correspondence: VMuchenje@hotmail.com

${ }^{2}$ Department of Livestock and Pasture Science, University of Fort Hare, Private Bag X1314, Alice 5700, South Africa

Full list of author information is available at the end of the article
}

plants are highly stress-tolerant plants which adapt easily to diverse climatic conditions [1].

According to the traditional healers of the study area, both plants can grow in all soil types. Cultivars of these plants prefer heavier soils such as loamy and gravely soil for quick growth, as this helps the plant to form dominant stands during dry seasons. The people of this region reported that these plants are effective against skin infection, sores, diabetes, infertility, high blood pressure and so on. Majority of them in the area are traditional healers (Sangomas) and rural dwellers, hence the use of medicinal plants for the treatment of certain diseases is very common. There is no doubt that some of these common diseases are usually caused by bacterial and viral pathogens, which definitely result to critical illness. Bacillus cereus, Enterococcus faecalis, Listeria 
monocytogenes, Staphylococcus aureus, Escherichia coli, Klebsiella pneumonia, Salmonella typhimurium and Pseudomonas aeruginosa are Gram-positive and negative bacteria and opportunistic pathogens of the human skin infection, toxic shock syndrome, urinary tract infections, gastroenteritis, and food poisoning [2].

Cympbopogon validis and plurinodis grow abundantly in this region and they are widely identified by the community dwellers as 'Irwashu' and 'Unukayo', respectively. As some of the rural dwellers are currently demanding for these plants because of their effectiveness, there is a dearth of information on their toxicity. Scientifically this study was therefore aimed at determining the plants' phytomedical activities as well as evaluating its safety in an effort to validate their folkloric use in the treatment of microbial infections.

\section{Methods}

\section{Plant extraction process}

After obtaining the ethics certificate approved by the University of Fort Hare's research ethics committee, the leaves of Cymbopogon validis and Cymbopogon plurinodis were collected in April, 2015 at 8 am in the morning in plastic bags. The botanical identification of these plants materials were confirmed by a botanist at the University of Fort Hare Institute. Voucher specimens were deposited at the institute's Herbarium.

A hundred and eighty-eight grams (188 g) of each fresh leaves were hydro-distilled separately in a clevenger's apparatus. Each samples were placed in a 5 -L round bottom flask fitted to a condenser. After adding $4 \mathrm{~L}$ distilled water, the cooling condenser was connected with the distillation assembly and heated to boiling. After 30 to $40 \mathrm{~min}$, boiling started; generated steam ruptured the cell walls of the leaves and released the oils present in the leaves. Distillation continued for $3 \mathrm{~h}$ for maximum oil recovery. The oil level in the separatory funnel was adjusted and maintained by varying the height of the outlet rubber tube. Once fixed, the excess water condensing in the seperatory funnel runs out spontaneously leaving accumulation of oil in the separatory funnel. After the distillation was over, each extracted oil was collected, filtered, and dried over anhydrous sodium sulphate $\left(\mathrm{Na}_{2} \mathrm{SO}_{4}\right)$. For the determination of the procedure yield, the solvent was evaporated using a rotatory vacuum evaporator (R-114; Buchi, New Castle, USA). Final yield of both plants' oil extracts were weighed and kept in separate clean bottles of known mass, labelled C. validis and C. plurinodis. The sensory characteristics of the essential oil from both plants were visualised based on their colour, clarity, aroma and odour intensity (Table 1). The yield obtained was calculated as follows: Mass of plant material distilled $(\mathrm{g})=\mathrm{X}$; Mass of empty bottle $(\mathrm{g})=\mathrm{A}$; Mass of bottle + oil
$(\mathrm{g})=\mathrm{B}$; Mass of oil $(\mathrm{g})=(\mathrm{B}-\mathrm{A})$; Percentage $(\%)$ yield = $[(\mathrm{B}-\mathrm{A}) \div \mathrm{X}] \times 100$. The resulting essential oils were stored at $-20{ }^{\circ} \mathrm{C}$ prior to further analysis.

\section{Phytochemical analysis}

The phytochemical analysis of C. validis and C. plurinodis essential oil were determined by gas chromatographymass spectroscopy instrument (HP 6890) with a mass selective detector (HP-5973). Identification of the chemical components of each essential oil was accomplished by marching their mass spectra and retention indices with those of the Wiley 275 library [3]. The quantity of compounds was calculated by integrating the peak areas of the spectrograms. A needle with $1.0 \mu \mathrm{l}$ sample oil (essential oils tested) was inserted directly into the inlet of the Gas Chromatograph. The initial temperature $70{ }^{\circ} \mathrm{C}$, maximum temperature $325{ }^{\circ} \mathrm{C}$, equilibration time $3 \mathrm{~min}$, ramp $4{ }^{\circ} \mathrm{C} /$ min, final temperature $240{ }^{\circ} \mathrm{C}$; inlet: split less, initial temperature $220^{\circ} \mathrm{C}$, pressure $8.27 \mathrm{psi}$, purge flow $30 \mathrm{ml} /$ min, purge time $0.20 \mathrm{~min}$. Helium was used as a carrier gas at a flow rate of $8.27 \mathrm{psi}$; the mass spectrometer was operated at $70 \mathrm{eV}$, column capillary, $30 \mathrm{~m} \times 0.25 \mathrm{~mm}$ ID fused silica column coated with DB-1: film thickness $0.25 \mu \mathrm{m}$, initial flow $0.7 \mathrm{ml} / \mathrm{min}$, average velocity $32 \mathrm{~cm} / \mathrm{s}$; MS: El method at $70 \mathrm{eV}$. The scan time was $0.36 \mathrm{~s}$ with internal scan delay of $0.05 \mathrm{~s}$ and mass range $40-300$. Compounds identified in the samples were confirmed by comparing their GC retention times with standards through a comparison of the mass spectra with available NIST Library 1 and with the softcopy results of the GCMS Turbo Mass. Quantification of essential oil components, expressed in relative percentage on the total area of identified chromatogram, was carried out by peak area normalization measurements.

\section{Bacterial strains and chemicals}

Four strains of Gram-positive bacteria: Bacillus cereus (ATCC \#10702), Enterococcus faecalis (ATCC \#29212), Listeria monocytogenes (ATCC \#12022), Staphylococcus aureus (ATCC \#6538) and four Gram-negative bacteria: Escherichia coli (ATCC \#8739), Klebsiella pneumonia (ATCC \#4354), Salmonella typhimurium (ATCC \#13311) and Pseudomonas aeroginosa (ATCC \#19582) were obtained from the Department of Biochemistry and Microbiology, University Fort Hare, Alice, South Africa. Ciprofloxacin, $p$-iodonitrotetrazolium violet ( $p$-INT) and dimethylsulfoxide (DMSO) were purchased from SigmaAldrich (St. Louis, Missouri).

\section{Cell line growth and maintenance}

The human Chang liver cell line used in this study was donated by Professor Maryna van de Venter from Nelson Mandela Metropolitan University, South Africa. Briefly, 
Table 1 Sensory evaluation and final percentage oil yield of Cymbopogon validis and Cymbopogon plurinodis

\begin{tabular}{|c|c|c|c|c|c|}
\hline \multicolumn{3}{|l|}{ C. Validis } & \multicolumn{3}{|l|}{ C. Plurinodis } \\
\hline Characteristics & Observation & Essential oil & Characteristics & Observation & Essential oil \\
\hline Colour & Yellowish & & Colour & Yellowish & 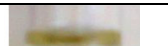 \\
\hline Clarity & $\begin{array}{l}\text { Slightly } \\
\text { murky }\end{array}$ & & Clarity & $\begin{array}{l}\text { Slightly } \\
\text { murky }\end{array}$ & \\
\hline Aroma & Rose-aroma & & Aroma & $\begin{array}{l}\text { Turpentine- } \\
\text { like }\end{array}$ & \\
\hline $\begin{array}{l}\text { Odour } \\
\text { intensity }\end{array}$ & $\begin{array}{l}\text { Slightly } \\
\text { strong }\end{array}$ & & $\begin{array}{l}\text { Odour } \\
\text { intensity }\end{array}$ & Strong & \\
\hline $\begin{array}{l}\text { Final } \\
\text { Percentage oil } \\
\text { yield }\end{array}$ & $1.11 \%$ & & $\begin{array}{l}\text { Final } \\
\text { percentage oil } \\
\text { yield }\end{array}$ & $0.54 \%$ & \\
\hline
\end{tabular}

vials containing cells were taken from liquid nitrogen stocks and thawed in a water bath of approximately $37^{\circ} \mathrm{C}$, and then transferred to a $25 \mathrm{~mm}^{3}$ culture flask (TPP, Switzerland). A I ml thawed cell stock was diluted with $9 \mathrm{ml}$ pre-warmed Dulbecco's minimum essential medium (DMEM) containing 10\% fetal bovine serum (FBS). The cells were incubated in a $37{ }^{\circ} \mathrm{C}$ humidified incubator (Shel Lab, USA), $5 \% \mathrm{CO}_{2}$ for multiplication and adherence. Maintenance of cells was achieved by splitting the cells until the desired cell number and confluence was reached.

\section{Preparation of bacterial inoculums}

The above bacteria strains were selected based on their public health disease-causing food borne poisons, bloody diarrhoea, anaemia, meningitis, pneumonia, etc. The inoculums of the test bacteria were prepared using the colony suspension method [4]. Colonies picked from $24 \mathrm{~h}$ old cultures grown on nutrient agar were used to make suspensions of the test organisms in saline solution to an optical density of approximately 0.1 at $600 \mathrm{~nm}$. The suspension was then diluted $1: 100 \mathrm{v} / \mathrm{v}$ by transferring $0.1 \mathrm{ml}$ of the bacteria suspension to $9,9 \mathrm{ml}$ of sterile Mueller Hinton broth. The cell turbidity was assessed spectroscopically in comparison to that of the 0.5 McFarland standards (approximately $1.5 \times 10^{8} \mathrm{cfu} / \mathrm{ml}$ ) before being used for antibacterial assays [5].

\section{Antibacterial activity assay}

Antibacterial assay was determined using a micro-well dilution method [6]. Each plant's essential oil was dissolved in DMSO and then in Mueller Hinton broth to reach a final concentration of $500.0 \mu \mathrm{g} / \mathrm{ml}$. Two-fold serial dilutions were made in a concentration range from 7.8 to $500.0 \mu \mathrm{g} / \mathrm{ml}$ in sterile test tubes containing Mueller Hinton broth. The 96-well plates were prepared by dispensing into each well $95 \mu \mathrm{l}$ of Mueller Hinton broth, $5 \mu \mathrm{l}$ of the bacteria inoculum and a $100 \mu \mathrm{l}$ from each serially diluted essential oil, transferred into six consecutive wells. The last well containing $195 \mu \mathrm{l}$ of Mueller Hinton broth without compound and $5 \mu \mathrm{l}$ of the inoculum on each strip was used as a negative control. The final volume in each well was $200 \mu \mathrm{l}$. Antibiotics of ciprofloxacin at same concentration range of 7.8 to $500.0 \mu \mathrm{g} / \mathrm{ml}$ was also prepared in Mueller Hinton broth and used as standard drug for positive control. Contents of each well were mixed on a plate shaker at $300 \mathrm{rpm}$ for $20 \mathrm{~s}$ prior to incubation at $37^{\circ}$ $\mathrm{C}$ for $24 \mathrm{~h}$. Each experiment was tested in triplicate. As an indicator of micro organism growth, $40 \mu \mathrm{l}$ of $p$-iodonitrotetrazolium violet $(p$-INT) dissolved in water were added to the wells and incubated at $37{ }^{\circ} \mathrm{C}$ for $30 \mathrm{~min}$ [7]. The colourless tetrazolium dye acts as an electron acceptor and is reduced to a red-coloured formazan product by biologically active organisms (Fig. 1). Where microbial growth was inhibited, the solution in the well remained clear after incubation with INT and was taken as its minimum inhibitory concentration (MIC ${ }^{\mathrm{INT}}$ ) at which no red colour occur. This was confirmed by plating $5 \mu$ l samples from clear wells on Mueller Hinton agar medium. The determinations of MIC values were done in triplicate.

\section{Cytotoxicity assay}

Toxicological effect of both plant extracts were evaluated on human Chang liver cell lines using microculture CellTiter-Blue viability (Promega, USA) assay. Briefly, 96-well microplates were seeded accurately, with $100 \mu \mathrm{l}$ DMEM + high glucose, L-glutamine and sodium pyruvate (Thermo Scientific, South Logan, Utah, USA) containing $3.0 \times 10^{3}$ cells in suspension and incubated in a $\mathrm{CO}_{2}$ incubator regulated at $37{ }^{\circ} \mathrm{C}$ and $5 \% \mathrm{CO}_{2}$. After $24 \mathrm{~h}$ incubation and attachment, the cells were treated with $1000,500,250,125,75,25$ and $5 \mu \mathrm{g} / \mathrm{ml}$ concentration of each extracts. Exactly $60 \mu \mathrm{m}$ of curcumin (Sigma-Aldrich, South Africa) was used as positive control and 0.1\% DMSO as negative control. After 24, 48, and $72 \mathrm{~h}$ of incubation, cell viability was determined by adding CellTiter-Blue as an indicator and further incubated for $4 \mathrm{~h}$. Fluorescence was read at 570/620 nm using Analytical \& Dignostic product Gen spectrophotometer (Bio Tek, USA). 

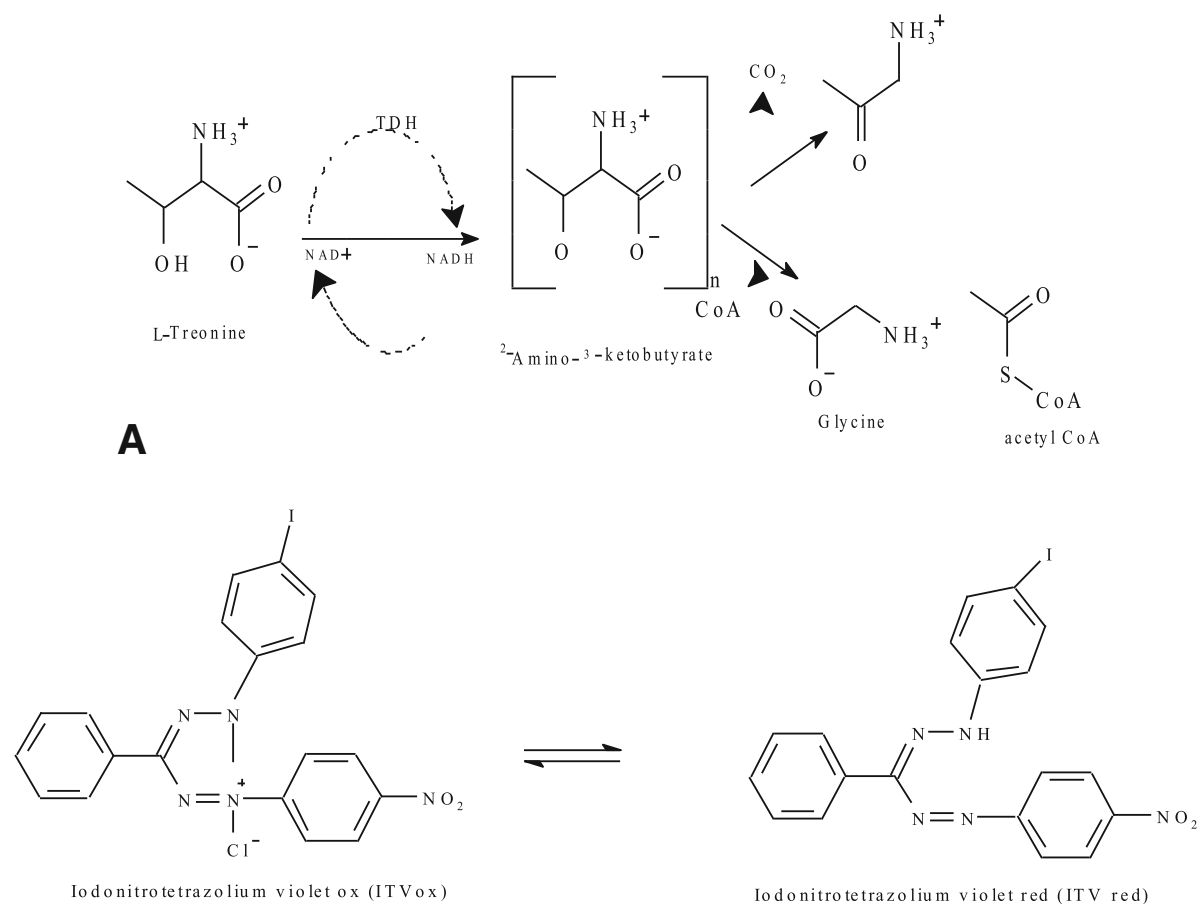

Iodonitro te trazolium violet red (ITV red)

B

Fig. 1 a Reaction pathways for the assay of threonine dehydrogenase, b INT, coupling reagent for the colorimetric assay

\section{Results and discussion}

\section{Sensory evaluation and essential oil yield}

The first steps of plant essential oil testing usually begin with sensory evaluation. This help to evaluate the colour, clarity and odour intensity of any essential oil. These attributes are usually stored in their security cells, such as cavities, glandular trichomes or epidermic cells [8]. The results of the sensory evaluation and final percentage oil yield from Cympbopogon validis and Cympbopogon plurinodis is shown in Table 1.

Common characteristics observed from the essential oil extracts from both plants were yellowish in colour and slightly murky. The odour of $C$. valids was having a rose-like aroma and slightly strong, while that of C. plurinodis was turpentine-like, and very strong. Their final percentage of the essential oil yields were $0.54 \%$ and $1.11 \%$, respectively.

\section{Chemical compounds of the essential oil}

The Gas chromatography/mass spectroscopy analysis of the plants' essential oils showed varied occurrence of volatile compounds which are mostly found in food and medicine. Hydro-distilled analysis of $C$. validis resulted in the identification of 80 phyto-compounds and the total amount of their calculated peak area percentage was $87.03 \%$ (Table 2). On the other hand, about 70 phytocompounds of $C$. plurinodis were identified and the total peak area percentage amounted to $81.47 \%$ (Table 3 ). The major compounds of Cymbopogon validis essential oil, based on the mass spectra peak areas were identified as Linalyl alcohol (18.9\%), 2-Nephthalenemethanol (6.67\%), Longifolene (6.53\%), Cubedol (6.08\%), $\beta$-Myrcene (4.85\%), Santolina triene $(4.60 \%)$, geraniol $(2.68 \%)$, and 4 -epicubedol (2.41\%), while the main compounds of Cymbopogon plurinodis were characterized as 3-Cyclohexane-1-ol (13.58\%), Nerolidol (13.6\%), 2-Carene (12.6\%), $z$-Selinene (8.50\%), $\beta$-Myrcene (4.73\%) and D-Limonene (3.65\%) (Tables 2 and 3).

Generally, the phyto-constituents present in any plant essential oil are differentiated by their primary chemical groups of terpenic hydrocarbons, such as monoterpenes, sequiterpenes, diterpenes, aldehydes, esters, phenols, ketones and alcohols. All terpenes are essential building blocks in plant biochemistry and majority of them are used for different purposes in the pharmaceuticals, cosmetics, and food preservatives [9]. Some major components of terpene hydrocarbons such as, Carvcrol (4.1\%),

-Terpinene (39.26\%) and Thymol (25.16\%) from the essential oil of Satureja thymbra were found to have potent antibacterial inhibitory activity against all strains tested $[10,11]$. In this study, the various chemical compounds from the essential oil of C. validis and C. plurinodis were profiled as monoterpenes $\left(C_{10}\right)$, oxygenated monoterpenes $\left(C_{10} \mathrm{O}\right)$, sesquiterpenes $\left(C_{15}\right)$, oxygenated 
Table 2 Chemical compounds of Cymbopogon validis leaf

\begin{tabular}{lllll}
\hline Peak Phyto-compounds & Rt & Area & Library & Chemical \\
number & (mins) $\%$ & quality & formulas \\
& match &
\end{tabular}

\begin{tabular}{|c|c|c|c|c|c|}
\hline & & & & (\%) & \\
\hline 1 & $\begin{array}{l}\text { Cyclopentanol, 3- } \\
\text { methylene }\end{array}$ & 3.26 & 0.01 & 70 & $\mathrm{C}_{5} \mathrm{H}_{10} \mathrm{O}$ \\
\hline 2 & 2-Hexenal & 3.33 & 0.03 & 92 & $\mathrm{C}_{6} \mathrm{H}_{12} \mathrm{O}$ \\
\hline 3 & Trans-7-methyl-3-octene & 3.40 & 0.01 & 72 & $\mathrm{C}_{9} \mathrm{H}_{18}$ \\
\hline 4 & 4-Heptanone & 3.46 & 0.02 & 87 & $\mathrm{C}_{7} \mathrm{H}_{14} \mathrm{O}$ \\
\hline 5 & 2-Heptanone & 3.58 & 0.06 & 74 & $\mathrm{C}_{7} \mathrm{H}_{14} \mathrm{O}$ \\
\hline 6 & 2-Heptanol & 3.64 & 0.06 & 83 & $\mathrm{C}_{7} \mathrm{H}_{16} \mathrm{O}$ \\
\hline 7 & 3-Carene & 3.93 & 0.19 & 94 & $\mathrm{C}_{10} \mathrm{H}_{16}$ \\
\hline 8 & a-Pinene & 4.01 & 0.37 & 96 & $\mathrm{C}_{10} \mathrm{H}_{16}$ \\
\hline 9 & Camphene & 4.15 & 0.68 & 97 & $\mathrm{C}_{10} \mathrm{H}_{16}$ \\
\hline 10 & $\beta$-Phellandrene & 4.32 & 0.03 & 94 & $\mathrm{C}_{10} \mathrm{H}_{16}$ \\
\hline 11 & $\beta$-Myrcene & 4.41 & 4.85 & 86 & $\mathrm{C}_{10} \mathrm{H}_{16}$ \\
\hline 12 & Octanal & 4.49 & 0.01 & 86 & $\mathrm{C}_{8} \mathrm{H}_{16} \mathrm{O}$ \\
\hline 13 & a-Phellandrene & 4.57 & 0.03 & 64 & $\mathrm{C}_{10} \mathrm{H}_{16}$ \\
\hline 14 & Allylidenecyclohexane & 4.61 & 0.05 & 45 & $\mathrm{C}_{9} \mathrm{H}_{14}$ \\
\hline 15 & 4-Carene & 4.67 & 0.01 & 98 & $\mathrm{C}_{10} \mathrm{H}_{16}$ \\
\hline 16 & Santolina triene & 4.78 & 4.60 & 86 & $\mathrm{C}_{10} \mathrm{H}_{16}$ \\
\hline 17 & $\beta$-Ocimene & 4.87 & 2.72 & 94 & $\mathrm{C}_{10} \mathrm{H}_{16}$ \\
\hline 18 & 4-Methy-1,5-Heptadiene & 4.92 & 0.11 & 64 & $\mathrm{n} / \mathrm{a}$ \\
\hline 19 & $\gamma$-Terpinene & 5.00 & 0.02 & 97 & $\mathrm{C}_{10} \mathrm{H}_{18} \mathrm{O}$ \\
\hline 20 & Hexanethioic acid & 5.07 & 2.53 & 91 & $\mathrm{C}_{6} \mathrm{H}_{12} \mathrm{OS}$ \\
\hline 21 & $\begin{array}{l}\text { Ethyl 2-(5-methyl-5- } \\
\text { vinyltetrahydrofuran-2- } \\
\text { yl)propan-2-yl carbonate }\end{array}$ & 5.13 & 1.44 & 91 & $\mathrm{n} / \mathrm{a}$ \\
\hline 22 & Linalyl alcohol & 5.37 & 18.9 & 92 & $\mathrm{C}_{10} \mathrm{H}_{18} \mathrm{O}$ \\
\hline 23 & $\begin{array}{l}\text { 4-chlorobenzoic acid, 4- } \\
\text { hexadecyl ester }\end{array}$ & 5.45 & 0.11 & 90 & $\mathrm{C}_{23} \mathrm{H}_{37} \mathrm{ClO}_{2}$ \\
\hline 24 & 2,4,6-Octatriene & 5.54 & 0.65 & 97 & $\mathrm{C}_{8} \mathrm{H}_{12}$ \\
\hline 25 & 5-Tetradecen-3-yne & 5.63 & 0.07 & 72 & $\mathrm{C}_{14} \mathrm{H}_{24}$ \\
\hline 26 & Citronellal & 5.72 & 0.77 & 96 & $\mathrm{C}_{10} \mathrm{H}_{20} \mathrm{O}$ \\
\hline 27 & 3-Hexadecene & 5.82 & 0.09 & 83 & $\mathrm{C}_{16} \mathrm{H}_{32}$ \\
\hline 28 & Endo-Borneol & 5.92 & 0.69 & 97 & $\mathrm{C}_{10} \mathrm{H}_{18} \mathrm{O}$ \\
\hline 29 & Terpinen-4-ol & 5.99 & 0.08 & 96 & $\mathrm{C}_{10} \mathrm{H}_{18} \mathrm{O}$ \\
\hline 30 & a-Terpineol & 6.07 & 0.59 & 87 & $\mathrm{C}_{10} \mathrm{H}_{18} \mathrm{O}$ \\
\hline 31 & Disparlure & 6.20 & 0.03 & 64 & $\mathrm{C}_{19} \mathrm{H}_{38} \mathrm{O}$ \\
\hline 32 & Citronellol & 6.26 & 1.47 & 98 & $\mathrm{C}_{10} \mathrm{H}_{18} \mathrm{O}$ \\
\hline 33 & $\begin{array}{l}\text { 2,6-Octadien-1-ol, 3,7- } \\
\text { dimethylene }\end{array}$ & 6.33 & 0.04 & 86 & $\mathrm{C}_{10} \mathrm{H}_{16} \mathrm{O}$ \\
\hline 34 & Citral & 6.40 & 0.16 & 95 & $\mathrm{C}_{10} \mathrm{H}_{16} \mathrm{O}$ \\
\hline 35 & Geraniol & 6.48 & 2.68 & 95 & $\mathrm{C}_{10} \mathrm{H}_{18} \mathrm{O}$ \\
\hline 36 & 4-Undecanone & 6.60 & 0.94 & 95 & $\mathrm{C}_{11} \mathrm{H}_{22} \mathrm{O}$ \\
\hline 37 & Trans-Geranylgeraniol & 6.74 & 0.01 & 68 & $\mathrm{C}_{20} \mathrm{H}_{34} \mathrm{O}$ \\
\hline 38 & Bornyl acetate & 6.78 & 0.06 & 99 & $\mathrm{C}_{12} \mathrm{H}_{20} \mathrm{O}_{2}$ \\
\hline 39 & $d$-Nonalactone & 6.85 & 0.05 & 38 & $\mathrm{C}_{9} \mathrm{H}_{16} \mathrm{O}_{2}$ \\
\hline
\end{tabular}

Table 2 Chemical compounds of Cymbopogon validis leaf (Continued)

\begin{tabular}{|c|c|c|c|c|c|}
\hline$\overline{40}$ & 2-Methoxy-4-vinylphenol & 6.95 & 0.03 & 38 & $\mathrm{C}_{9} \mathrm{H}_{10} \mathrm{O}_{2}$ \\
\hline 41 & 1-Ethynylcyclopentanol & 7.05 & 0.01 & 64 & $\mathrm{C}_{7} \mathrm{H}_{10} \mathrm{O}$ \\
\hline 42 & $\begin{array}{l}\text { 2,6-Octadiene,2,6- } \\
\text { dimethylene }\end{array}$ & 7.14 & 0.99 & 97 & $\mathrm{C}_{10} \mathrm{H}_{16}$ \\
\hline 43 & a-Cubebene & 7.24 & 0.11 & 99 & $\mathrm{C}_{15} \mathrm{H}_{24}$ \\
\hline 44 & Geranyl acetate & 7.35 & 1.97 & 90 & $\mathrm{C}_{12} \mathrm{H}_{20} \mathrm{O}_{2}$ \\
\hline 45 & Epizonarene & 7.42 & 0.31 & 98 & $\mathrm{C}_{15} \mathrm{H}_{24}$ \\
\hline 46 & $\begin{array}{l}\text { Cyclohexane, 1-ethenyl-1- } \\
\text { methyl-2,4-bis-1- } \\
\text { methylethenyl. }\end{array}$ & 7.54 & 1.26 & 99 & $\mathrm{n} / \mathrm{a}$ \\
\hline 47 & Formic acid & 7.65 & 0.04 & 55 & $\mathrm{CH}_{2} \mathrm{O}_{2}$ \\
\hline 48 & Caryophyllene & 7.79 & 0.64 & 98 & $\mathrm{C}_{15} \mathrm{H}_{24}$ \\
\hline 49 & a-Cubebene & 7.97 & 0.81 & 70 & $\mathrm{C}_{15} \mathrm{H}_{24}$ \\
\hline 50 & $\begin{array}{l}\text { Epi- } \\
\text { Bicyclosesquiphellandrene }\end{array}$ & 8.05 & 0.05 & 87 & $\mathrm{C}_{15} \mathrm{H}_{24}$ \\
\hline 51 & Y-Muurolene & 8.10 & 0.65 & 83 & $\mathrm{C}_{15} \mathrm{H}_{24}$ \\
\hline 52 & a-Muurolene & 8.14 & 1.66 & 99 & $\mathrm{C}_{15} \mathrm{H}_{24}$ \\
\hline 53 & 4-epi-cubedol & 8.52 & 2.41 & 93 & $\mathrm{C}_{15} \mathrm{H}_{26} \mathrm{O}$ \\
\hline 54 & Nephthalene & 8.31 & 0.96 & 96 & $\mathrm{C}_{10} \mathrm{H}_{8}$ \\
\hline 55 & Cubedol & 8.39 & 6.08 & 95 & $\mathrm{C}_{15} \mathrm{H}_{26} \mathrm{O}$ \\
\hline 56 & Di-epi-alpha-cedrene & 8.47 & 0.57 & 87 & $\mathrm{n} / \mathrm{a}$ \\
\hline 57 & Guaia-1,11-diene & 8.56 & 2.05 & 46 & $\mathrm{C}_{15} \mathrm{H}_{24}$ \\
\hline 58 & Epizonarene & 8.61 & 0.22 & 81 & $\mathrm{C}_{15} \mathrm{H}_{24}$ \\
\hline 59 & Cyclohexane & 8.64 & 0.44 & 30 & $\mathrm{C}_{6} \mathrm{H}_{12}$ \\
\hline 60 & Gleenol & 8.77 & 0.54 & 97 & $\mathrm{C}_{15} \mathrm{H}_{26} \mathrm{O}$ \\
\hline 61 & 2-Naphthalenemethanol & 8.96 & 6.67 & 86 & $\mathrm{C}_{11} \mathrm{H}_{10} \mathrm{O}$ \\
\hline 62 & Longifolene & 9.08 & 6.53 & 93 & $\mathrm{C}_{15} \mathrm{H}_{24}$ \\
\hline 63 & Hinesol & 9.19 & 2.14 & 93 & $\mathrm{C}_{15} \mathrm{H}_{26} \mathrm{O}$ \\
\hline 64 & Agarospirol & 9.25 & 0.37 & 98 & $\mathrm{C}_{15} \mathrm{H}_{26} \mathrm{O}$ \\
\hline 65 & Alloaromadendrene & 9.42 & 0.37 & 53 & $\mathrm{C}_{15} \mathrm{H}_{24}$ \\
\hline 66 & Isolongifolene & 9.53 & 0.46 & 38 & $\mathrm{C}_{15} \mathrm{H}_{24}$ \\
\hline 67 & $\begin{array}{l}\text { Ethyladamantane-1- } \\
\text { carboxylate }\end{array}$ & 9.70 & 0.19 & 38 & $\mathrm{C}_{13} \mathrm{H}_{20} \mathrm{O}_{2}$ \\
\hline 68 & $\beta$-Humulene & 9.77 & 0.12 & 44 & $\mathrm{C}_{15} \mathrm{H}_{24}$ \\
\hline 69 & Isocaryophillene & 9.87 & 0.12 & 56 & $\mathrm{C}_{15} \mathrm{H}_{24}$ \\
\hline 70 & Ledol & 10.0 & 0.05 & 51 & $\mathrm{C}_{15} \mathrm{H}_{26} \mathrm{O}$ \\
\hline 71 & Acetic acid & 10.1 & 2.69 & 38 & $\mathrm{C}_{2} \mathrm{H}_{4} \mathrm{O}_{2}$ \\
\hline 72 & a-Guaiene & 10.2 & 0.03 & 52 & $\mathrm{C}_{15} \mathrm{H}_{24}$ \\
\hline 73 & 4-Fluorobenzoic acid & 10.4 & 0.02 & 62 & $\mathrm{C}_{7} \mathrm{H}_{5} \mathrm{FO}_{2}$ \\
\hline 74 & Octasiloxane & 11.3 & 0.01 & 41 & $\mathrm{H}_{6} \mathrm{OSi} 2$ \\
\hline 75 & 2-Nonadecanone & 12.2 & 0.01 & 64 & $\mathrm{C}_{19} \mathrm{H}_{38} \mathrm{O}$ \\
\hline 76 & Hexamethylene & 13.2 & 0.01 & 43 & $\mathrm{C}_{7} \mathrm{H}_{14}$ \\
\hline 77 & Arsenous acid & 13.8 & 0.04 & 43 & $\mathrm{H}_{3} \mathrm{AsO}_{3}$ \\
\hline 78 & Diethyl ether & 14.2 & 0.05 & 38 & $\mathrm{C}_{4} \mathrm{H}_{10} \mathrm{O}$ \\
\hline 79 & 3-hexenyl ester & 14.6 & 0.02 & 38 & $\mathrm{C}_{10} \mathrm{H}_{16}$ \\
\hline 80 & 4- hexadecyl ester & 14.8 & 0.11 & 43 & $\mathrm{C}_{16} \mathrm{H}_{33}$ \\
\hline
\end{tabular}


Table 2 Chemical compounds of Cymbopogon validis leaf (Continued)

\begin{tabular}{ll}
\hline Total amount of compounds & 87.03 \\
Monoterpene hydrocarbon & 16.74 \\
Oxygenated monoterpenes & 31.43 \\
Sesquiterpene hydrocarbon & 14.26 \\
Oxygenated sesquiterpene & 11.6 \\
Oxygenated diterpene & 0.01 \\
Aldehydes & 6.81 \\
Fatty acids & 2.78 \\
Others & 3.40 \\
\hline
\end{tabular}

sesquiterpenes $\left(\mathrm{C}_{15} \mathrm{O}\right)$, diterpenes $\left(\mathrm{C}_{20}\right)$, oxygenated diterpenes $\left(\mathrm{C}_{20} \mathrm{O}\right)$, aldehydes $(\mathrm{CHO})$ and fatty acids $(\mathrm{COH})$. From the chemical formulas of each $C$. validis phytocompounds in Table 2, the total amount of $\mathrm{C}_{10}$ monoterpenes hydrocarbons was $25.49 \%$. Highest peak library match of compounds were $\beta$-Myrcene (4.85\%) and Santolina triene (4.60\%). On the hand, the total amount of C. plurinodis $\mathrm{C}_{10}$ monoterpenes hydrocarbons resulted to $16.74 \%$. Highest peak values were 2-Carene (12.6\%), $\beta$ - Myrcene (4.73\%) and D-Limonene (3.65\%) (Table 3).

The total amount of $\mathrm{C}_{10} \mathrm{O}$ hydrocarbons which signifies the oxygenated monoterpenes was calculated to be $18.37 \%$ for C. validis essential oil. For C. plurinodis essential oil, the total amount of oxygenated monoterpenes was found lower with $31.43 \%$. Linalyl alcohol (18.9\%) and 3-Cyclohexane-1-ol (13.58\%) were their highest peak match, respectively (Tables 2 and 3). The inhibitory activities of the essential oil extracted from Ridolfia segetum, Oenanthe crocata and Santolina chamaecyparissus, with their chemical constituents comprising of $\beta$ Myrcene, Santolina triene and Limonene have been reported active against HIV-1 reverse transcriptase, human tumor, oxidant and inflammatory activities [12, 13]. Limonene itself has immune-stimulatory, analgesic and anaesthetic properties [14]. Immune modulation and antiproliferative effects of limonene's anti-cancer activity have also been reported [14]. Manufacturing industries use 3-Cyclohexane and $\beta$-Myrcene as additives in producing perfumes, pesticides, polyvinyl, and nitrocellulose resins [15]. Over 200 families of Lamiaceae plants are known to produce large amounts of chemicals of Linalyl alcohol and 2-Nephthalenol, which are used as a scent in $60-80 \%$ of perfumed hygiene products, oxidizing colouring agents and cleaning agents including soaps, detergents, shampoos and lotions [16]. Medically, Linalyl alcohol therapy has been studied to reduced serum cortisol and improved the coronary flow velocity reserve (CFVR) in healthy men [17]. The findings revealed that Linalyl alcohol has a relaxation activity to relieve back pain, muscle stiffness, and cramps [17]. Another common downstream product of Linalyl alcohol is Vitamin $\mathrm{E}$, a rich compound that naturally reduces cholesterol and the risk of developing cancer [18].

The total amount of $\mathrm{C}_{15}$ sesquiterpene hydrocarbons of C. validis essential oil gave $13.20 \%$, compared to $C$. plurinodis sesquiterpene hydrocarbons, which gave $14.26 \%$. Longifolene $(6.53 \%)$ and $z$-Selinene $(8.50 \%)$ were found to be their highest peak match, respectively. The calculated total amount of C. plurinodis oxygenated sesquiterpenes was $11.6 \%$, while that of $C$. validis was 17.66\%. Nerolidol $2(13.6 \%)$ and 4-epi-cubedol $(2.41 \%)$ were observed as their highest values respectively. Essential oil containing sesquiterpenes are used to treat inflammatory and allergic infections [19]. Research has found that people who consistently use essential oil containing sesquiterpenes, have a higher level of resistance to illness than the average person. Further investigation revealed that if such an individual peradventure falls ill, he or she showed a $60-70 \%$ recovery than those not using essential oil products [20, 21]. Longifolene and $z$-Selinene are some of the most abundant sesquiterpene hydrocarbons naturally occurring in $P$. longifolia, $P$. roxburghii and $P$. sylyestris. They are used as chemicals in perfumery industry owing to the woody odour of their chemically modified forms [22]. Both compounds have potent antioxidant and anti-inflammatory and anticancer properties. They offer assistance to a variety of metabolic and health problems, helps in weight management, liver detoxification enzymes, improve indigestion and sluggish bowel [23]. 4-epi-cubedol and Nerolidol are mainly found in some specific plants such as neroli, ginger, jasmine, lavender, tea tree, and lemon grass. They are mainly used as a food-flavoring agent and perfumery [24]. These compounds are currently under testing as a skin penetration enhancer for the transdermal delivery of therapeutic drugs [25]. Nerolidol works as a specific compound that female mites use to attract males for mating, in order words, Nerolidol is considered safe for humans and the environment.

Trans-geranylgeraniol (0.01\%) Phytol (0.05\%) and Epiglobulol $(0.09 \%)$ were the major concentrated oxygenated diterpenes $\left(\mathrm{C}_{20} \mathrm{O}\right)$ detected in both plants essential oil of C. validis and C. plurinodis. These oxygenated compounds have the ability to inhibit microbial causing infections [26]. Phytol administered to mice at increasing dose responds of $25,50,10$, and $200 \mathrm{mg} / \mathrm{kg}$ showed pronounced anti-nociceptive effects in the nociception models used $[27,28]$. In vitro antioxidant activity of phytol demonstrated a strong effect against hydroxyl radicals and nitric oxide [27, 28]. A total content of aldehydes $(\mathrm{CHO})$ present in C. validis was $6.81 \%$. Of these, Nephthalenemethanol content showed the highest peak area of $6.67 \%$. In contrast, 4 -Nonanol $(0.25 \%)$ was the highest peak value found in C. plurinodis aldehydes, 
Table 3 Chemical compounds of Cymbopogon plurinodis leaf

\begin{tabular}{|c|c|c|c|c|}
\hline $\begin{array}{l}\text { GC } \\
\text { peak } \\
\text { number }\end{array}$ & Phyto-compounds & $\begin{array}{l}\text { Rt } \\
\text { (mins) }\end{array}$ & $\begin{array}{l}\text { Area } \\
\%\end{array}$ & $\begin{array}{l}\text { Library } \\
\text { quality } \\
\text { match }\end{array}$ \\
\hline
\end{tabular}

2-Hexenal

3-Carene

a-Pinene

3

2-Hexenal

Camphene

Benzaldehyde

$\beta$-Phellandrene

$\beta$-Myrcene

2-Carene

(+)-4-Carene

D-Limonene

$\beta$-Ocimene

1-Octanol

4-Nonanol

6-Undecanol

Linalyl acetate

Carveol

2-Cyclohexen-1-ol

3-Tetradecyn-1-ol

Camphor

Geraniol

1,3,5-Cycloheptadiene

endo-Borneol

Benzenamine,3-ethoxy

a-Terpineol

N-(2-Methyl-propenyl)-

pyrrolidin-2-one

Photocitral B

3-Cyclohexen-1-ol

Bornyl acetate

Hexanoic acid

Furan

9-Hexadecenoic acid

a-lonone

3-Nonen-1-ol

Geranyl acetate

Epizonarene

Cycloheptane, 4-

methylene-1-methyl-2-

(2-methyl-1-propen-1-

vinyl

$\begin{array}{ll}38 & \text { Nephthalene } \\ 39 & \text { Caryophyllene } \\ 40 & \text { a-Guaiene }\end{array}$

$\begin{array}{llll}7.62 & 0.12 & 78 & \mathrm{C}_{10} \mathrm{H}_{8} \\ 7.80 & 1.32 & 99 & \mathrm{C}_{15} \mathrm{H}_{24} \\ 7.85 & 0.31 & 38 & \mathrm{C}_{15} \mathrm{H}_{24}\end{array}$

Table 3 Chemical compounds of Cymbopogon plurinodis leaf (Continued)

\begin{tabular}{|c|c|c|c|c|c|}
\hline$\overline{41}$ & Isoledene & 7.93 & 0.10 & 97 & $\mathrm{C}_{15} \mathrm{H}_{24}$ \\
\hline 42 & 2,3-Octanedione & 7.97 & 0.23 & 58 & $\mathrm{C}_{8} \mathrm{H}_{14} \mathrm{O}_{2}$ \\
\hline 43 & Humulene & 8.01 & 0.29 & 97 & $\mathrm{C}_{15} \mathrm{H}_{24}$ \\
\hline 44 & $\beta$-Coapaene & 8.06 & 0.17 & 93 & $\mathrm{C}_{15} \mathrm{H}_{24}$ \\
\hline 45 & Alloaromadendrene & 8.10 & 0.75 & 80 & $\mathrm{C}_{15} \mathrm{H}_{24}$ \\
\hline 46 & 4-epi-cubedol & 8.38 & 2.22 & 99 & $\mathrm{C}_{15} \mathrm{H}_{26} \mathrm{O}$ \\
\hline 47 & Y-Muurolene & 8.47 & 0.95 & 43 & $\mathrm{C}_{15} \mathrm{H}_{24}$ \\
\hline 48 & Cycloprop (e) azulene & 8.50 & 0.83 & 70 & $\mathrm{C}_{10} \mathrm{H}_{8}$ \\
\hline 49 & Nerolidol 2 & 8.60 & 13.6 & 91 & $\mathrm{C}_{15} \mathrm{H}_{26} \mathrm{O}$ \\
\hline 50 & $\begin{array}{l}\text { Trifluoroacetyl-a- } \\
\text { fenchol }\end{array}$ & 8.79 & 0.94 & 87 & $\mathrm{n} / \mathrm{a}$ \\
\hline 51 & Caryophyllene oxide & 8.87 & 0.83 & 94 & $\mathrm{C}_{15} \mathrm{H}_{24} \mathrm{O}$ \\
\hline 52 & Ethanopentalen-4-ol & 9.09 & 0.90 & 38 & $\mathrm{n} / \mathrm{a}$ \\
\hline 53 & Tau-Cadinol & 9.13 & 0.76 & 86 & $\mathrm{C}_{15} \mathrm{H}_{26} \mathrm{O}$ \\
\hline 54 & Tau-Muurolol & 9.25 & 1.94 & 62 & $\mathrm{n} / \mathrm{a}$ \\
\hline 55 & $\boldsymbol{z}$-Selinene & 9.34 & 8.50 & 83 & $\mathrm{C}_{15} \mathrm{H}_{24}$ \\
\hline 56 & Trans- a-Bergamotene & 9.43 & 0.73 & 87 & $\mathrm{C}_{15} \mathrm{H}_{24}$ \\
\hline 57 & Pyrazole & 9.68 & 1.59 & 43 & $\mathrm{C}_{3} \mathrm{H}_{4} \mathrm{~N}_{2}$ \\
\hline 58 & $\beta$-Pinene & 9.76 & 0.37 & 58 & $\mathrm{C}_{10} \mathrm{H}_{16}$ \\
\hline 59 & $\beta$-Santalol & 9.82 & 0.38 & 53 & $\mathrm{n} / \mathrm{a}$ \\
\hline 60 & $\begin{array}{l}\text { Isoaromadendrene } \\
\text { epoxide }\end{array}$ & 10.0 & 0.02 & 91 & $\mathrm{C}_{15} \mathrm{H}_{24} \mathrm{O}$ \\
\hline 61 & Phytol acetate & 10.0 & 0.05 & 80 & $\mathrm{C}_{22} \mathrm{H}_{42} \mathrm{O}_{2}$ \\
\hline 62 & Epiglobulol & 10.1 & 0.09 & 41 & $\mathrm{C}_{20} \mathrm{H}_{40} \mathrm{O}$ \\
\hline 63 & Diethyl ether & 10.2 & 0.01 & 18 & $\mathrm{C}_{4} \mathrm{H}_{10} \mathrm{O}$ \\
\hline 64 & Shizukanolide & 11.2 & 0.23 & 44 & $\mathrm{C}_{15} \mathrm{H}_{18} \mathrm{O}_{2}$ \\
\hline 65 & 2-Ethylacridine & 11.4 & 0.01 & 25 & $\mathrm{C}_{15} \mathrm{H}_{13} \mathrm{~N}$ \\
\hline 66 & Heptasiloxane & 11.5 & 0.00 & 38 & $\mathrm{C}_{16} \mathrm{H}_{48} \mathrm{O}_{6} \mathrm{Si}_{7}$ \\
\hline 67 & Ester & 12.2 & 0.01 & 38 & $\mathrm{C}_{4} \mathrm{H}_{10} \mathrm{O}$ \\
\hline 68 & Octasiloxane & 13.0 & 0.00 & 87 & $\mathrm{H}_{6} \mathrm{OSi} 2$ \\
\hline 69 & Cyclotrisiloxane & 13.2 & 0.01 & 43 & $\mathrm{C}_{6} \mathrm{H}_{18} \mathrm{O}_{3} \mathrm{Si}_{3}$ \\
\hline 70 & 1,3,5-Hexatriene & 13.5 & 0.05 & 51 & $\mathrm{C}_{6} \mathrm{H}_{8}$ \\
\hline \multicolumn{2}{|c|}{ Total of compounds } & \multicolumn{4}{|l|}{81.47} \\
\hline \multicolumn{2}{|c|}{ Monoterpene hydrocarbon } & \multicolumn{4}{|l|}{25.49} \\
\hline \multicolumn{2}{|c|}{ Oxygenated monoterpenes } & \multicolumn{4}{|l|}{18.37} \\
\hline \multicolumn{2}{|c|}{ Sesquiterpene hydrocarbon } & \multicolumn{4}{|l|}{13.20} \\
\hline \multicolumn{2}{|c|}{ Oxygenated sesquiterpene } & \multicolumn{4}{|l|}{17.66} \\
\hline \multicolumn{2}{|c|}{ Oxygenated diterpene } & \multicolumn{4}{|l|}{0.14} \\
\hline \multicolumn{2}{|c|}{ Aldehydes } & \multicolumn{4}{|l|}{0.49} \\
\hline \multicolumn{2}{|c|}{ Fatty acids } & \multicolumn{4}{|l|}{0.09} \\
\hline \multicolumn{2}{|c|}{ Others } & \multicolumn{4}{|l|}{6.03} \\
\hline
\end{tabular}

from a total content of $0.49 \%$. Fatty acids $(\mathrm{COH})$ and their methyl esters present in both plant oils were present in smaller quantities, having total percentage of 
Table 4 Antibacterial activity of Cympbopogon validis and Cymbopogon plurinodis essential oils

\begin{tabular}{|c|c|c|c|c|}
\hline \multirow[t]{3}{*}{ Microorganisms } & \multirow{3}{*}{$\begin{array}{l}\text { Gram } \\
+/-\end{array}$} & \multirow{2}{*}{\multicolumn{2}{|c|}{$\frac{\text { MIC }^{\text {INT }} \text { values }(\mu \mathrm{g} / \mathrm{ml})}{\text { Essential oils }}$}} & \multirow[t]{3}{*}{ Ciprofloxacin } \\
\hline & & & & \\
\hline & & C. validis & C. plurinodis & \\
\hline Bacillus cereus & $\mathrm{G}+$ & 15.6 & 62.5 & 7.8 \\
\hline Enterococcus faecalis & $\mathrm{G}+$ & 62.5 & 31.2 & 62.5 \\
\hline Listeria monocytogenes & $\mathrm{G}+$ & 15.6 & 15.6 & 7.8 \\
\hline Staphylococcus aureus & $\mathrm{G}+$ & 15.6 & 125.0 & 15.6 \\
\hline Escherichia coli & G- & 62.5 & 500.0 & 15.6 \\
\hline Klebsiella pneumonia & G- & 500.0 & 500.0 & 31.2 \\
\hline Salmonella typhimurium & G- & 125.0 & 250.0 & 15.6 \\
\hline Pseudomonas aeroginosa & G- & 500.0 & $>500.0$ & 125.0 \\
\hline
\end{tabular}

0.09 and $2.78 \%$, respectively. Medicinally, these compounds are known to have anti-inflammatory, anticancer, anti-amoebic, allelopathic, free radical scavenging and other useful biological activities [29].

The antibacterial activities of Cymbopogon validis and Cymbopogon plurinodis essential oils were assayed in vitro by a broth micro-dilution method against eight pathogenic bacteria strains. According to the results, Cympbopogon validis essential oil was found to be active against all the pathogenic bacteria, except Klebsiella pneumonia and Pseudomonas aeroginosa which demonstrated weak inhibitory activity at the highest MIC concentration of $500 \mu \mathrm{g} / \mathrm{ml}$ (Table 4). The strongest antibacterial inhibitory activity was seen against Bacillus cereus, Listeria monocytogenes, and Staphylococcus aureus with MIC values of $15.6 \mu \mathrm{g} / \mathrm{ml}$ followed by Enterococcus faecalis and Escherichia coli MIC $62.5 \mu \mathrm{g} / \mathrm{ml}$, and then Salmonella typhimurium MIC at $125 \mu \mathrm{g} / \mathrm{ml}$. In contrast, the essential oil of Cympbopogon plurinodis also displayed inhibitory activity against all the bacteria except Pseudomonas aeroginosa. Significant growth reduction was observed only against Listeria monocytogenes with MIC value of $15.6 \mu \mathrm{g} / \mathrm{ml}$, followed by Enterococcus faecalis MIC $31.2 \mu \mathrm{g} / \mathrm{ml}$, Bacillus cereus at $62.5 \mu \mathrm{g} / \mathrm{ml}$, Staphylococcus aureus at $125.0 \mu \mathrm{g} / \mathrm{ml}$ and Salmonella typhimurium at $250 \mu \mathrm{g} / \mathrm{ml}$. Weak MIC inhibitory activity of $500 \mu \mathrm{g} / \mathrm{ml}$ was observed for Escherichia coli and Klebsiella pneumonia. The standard antibiotic ciprofloxacin showed potent inhibitory action against all bacteria tested. At the lowest MIC of $7.8 \mu \mathrm{g} / \mathrm{ml}$, ciprofloxacin was observed to reduce the growth of Bacillus cereus and Listeria monocytogenes, followed by Staphylococcus aureus, Escherichia coli and Salmonella typhimurium at MIC value of $15.6 \mu \mathrm{g} / \mathrm{ml}$. Ciprofloxacin MIC activity on Klebsiella pneumonia was $31.2 \mu \mathrm{g} / \mathrm{ml}$, Enterococcus faecalis at $62.5 \mu \mathrm{g} / \mathrm{ml}$ and Pseudomonas aeroginosa at $125.0 \mu \mathrm{g} / \mathrm{ml}$.

Both plant essential oils had little or no effect against the Gram-negative Pseudomonas aeroginosa due to its high level of intrinsic outer membrane barrier that is resistant to virtually all known antimicrobials and antibiotics [30]. Moreover, the results obtained are of a great importance particularly in the case of Bacillus cereus, Staphylococcus aureus and Listeria monocytogenes, which are well known for being resistant to a number of phytochemical compounds [31, 32]. Plant essential oils and extracts have been used for many thousands of years [33], especially in food preservation, pharmaceuticals, alternative medicine and natural therapies [30, 31]). Essential oils extracted from C. citratus, C. flexosus, C. naudus and $C$. winterianus exhibited activity against both Gram positive and Gram negative bacteria [34, 35]. Application of these essential oils on bacteria strains inhibited Acinetobacter baumanii, Enterococcus faecalis, Escherichia coli, Klebsiella pneumonia, Pseudomons aeruginosa, Salmonella typhimurium, Serratia marcescens and Staphylococcus aureus at the concentration of $1200 \mu \mathrm{g} / \mathrm{ml}$ to $<20,000 \mu \mathrm{g} /$ $\mathrm{ml}$ [36]. It has long been acknowledged that some plant essential oils exhibit antimicrobial properties, due to their monoterpene hydrocarbons, aldehydes and oxygenated monoterpenes. Compounds from these groups, such as Citronella, Camphene, Limonene, Sabinene, Geraniol and Phytol have been reported that they can diffuse into and damage cell membrane structures of organisms $[37,26]$.

\section{Cytotoxicological effect of the extracts on cell viability}

The liver is known as a unique organ and primary site of detoxification. Due to the important role of the liver

Table 5 Effective concentration and time interval on the percentage cell death

\begin{tabular}{|c|c|c|c|c|c|c|c|c|c|c|c|c|c|}
\hline \multicolumn{7}{|c|}{ Cymbopogon validis } & \multicolumn{7}{|c|}{ Cymbopogon plurinodis } \\
\hline \multirow[b]{2}{*}{ Percentile } & \multicolumn{3}{|c|}{ Probit } & \multicolumn{3}{|l|}{$\log (\text { dose })^{a}$} & \multirow[b]{2}{*}{ Percentile } & \multicolumn{3}{|c|}{ Probit } & \multicolumn{3}{|l|}{$\log (\text { dose })^{a}$} \\
\hline & $24 \mathrm{~h}$ & $48 \mathrm{~h}$ & $72 \mathrm{~h}$ & $24 \mathrm{~h}$ & $48 \mathrm{~h}$ & $72 \mathrm{~h}$ & & $24 \mathrm{~h}$ & $48 \mathrm{~h}$ & $72 \mathrm{~h}$ & $24 \mathrm{~h}$ & $48 \mathrm{~h}$ & $72 \mathrm{~h}$ \\
\hline 5 & 2.89 & 2.89 & 2.89 & $0.62 \pm 0.03$ & $0.67 \pm 0.01$ & $0.99 \pm 0.01$ & 5 & 2.11 & 2.11 & 2.11 & $0.74 \pm 0.03$ & $0.60 \pm 0.01$ & $0.18 \pm 0.01$ \\
\hline 25 & 3.67 & 3.67 & 3.67 & $0.76 \pm 0.03$ & $0.91 \pm 0.02$ & $1.67 \pm 0.01$ & 25 & 3.18 & 3.18 & 3.18 & $0.86 \pm 0.03$ & $0.77 \pm 0.02$ & $1.80 \pm 0.01$ \\
\hline 50 & 4.88 & 4.88 & 4.88 & $0.85 \pm 0.02$ & $1.88 \pm 0.01$ & $2.09 \pm 0.00$ & 50 & 4.01 & 4.01 & 4.01 & $0.18 \pm 0.02$ & $1.69 \pm 0.01$ & $2.11 \pm 0.0$ \\
\hline 75 & 5.13 & 5.13 & 5.13 & $2.05 \pm 0.01$ & $2.35 \pm 0.00$ & $2.77 \pm 0.01$ & 75 & 5.22 & 5.22 & 5.22 & $2.99 \pm 0.01$ & $2.75 \pm 0.00$ & $2.45 \pm 0.0$ \\
\hline 95 & 6.23 & 6.23 & 6.23 & $3.45 \pm 0.01$ & $3.64 \pm 0.01$ & $3.03 \pm 0.01$ & 95 & 6.45 & 6.45 & 6.45 & $3.95 \pm 0.01$ & $3.58 \pm 0.01$ & $3.33 \pm 0.0$ \\
\hline
\end{tabular}

${ }^{a}$ Antilog which gives lethal dose in $\mu \mathrm{g} / \mathrm{ml}$. Probit analysis NCSS 2007 used to determine log (dose), percentile and probit values 


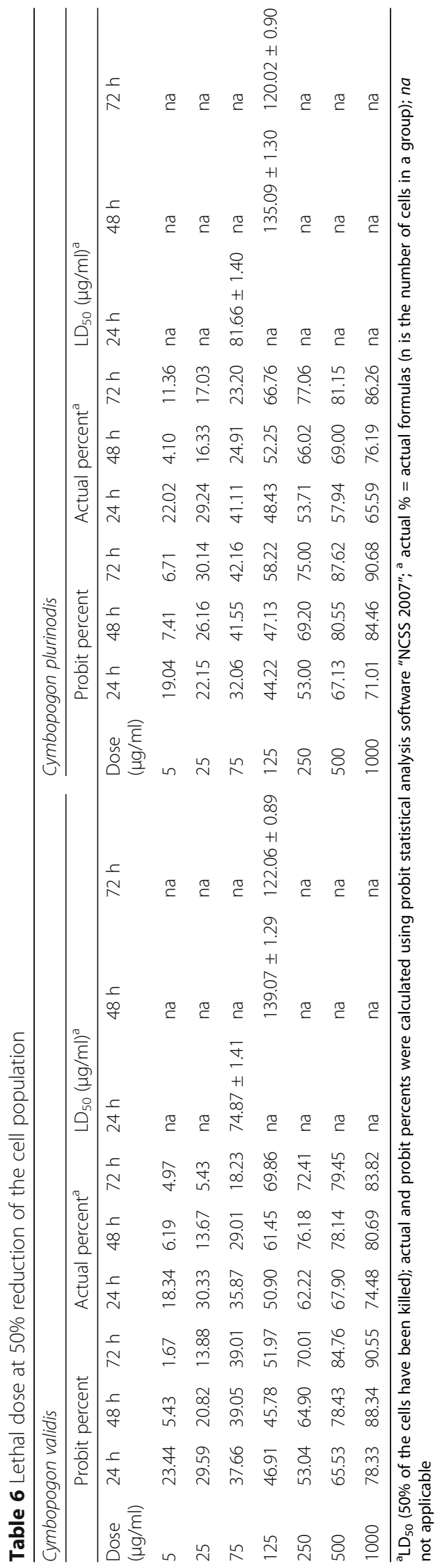


intense metabolism, it is likely to be prone to various disorders as a result of toxic chemicals [38]. Liver maintains the energy level and structural stability of the body. Therefore, any attempt to damage the liver either by any poisonous or harmful chemicals will definitely result to hepatotoxicity [38]. The toxicity of both plants extracts were tested to evaluate their effects on human Chang liver cells.

According to our cytotoxicity evaluation of both plants, we observed that at $5,25,50,75$, and 95 percentile of cell death after 24,48 , and $72 \mathrm{~h}$ of incubation, the C. validis extract dose activity ranged from $\log$ of $0.62 \pm 0.03$ to $0.99 \pm 0.01,0.76 \pm 0.03$ to $1.67 \pm 0.01$, $0.85 \pm 0.02$ to $2.09 \pm 0.00,2.05 \pm 0.01$ to $2.77 \pm 0.01$, and $3.45 \pm 0.01$ to $3.03 \pm 0.01$, respectively (Table 5). Cymbopogon plurinodis extract dose activity ranged from $\log$ of $0.74 \pm 0.03$ to $0.18 \pm 0.01,0.86 \pm 0.03$ to $1.80 \pm 0.01,0.18 \pm 0.02$ to $2.11 \pm 0.00,2.99 \pm 0.01$ to $2.45 \pm 0.01,3.95 \pm 0.01$ to $3.33 \pm 0.01$, respectively. Lethal dose of $C$. validis at $50\left(\mathrm{LD}_{50}\right)$ showed $74.87 \pm 1.41$,
$139.07 \pm 1.29$, and $122.06 \pm 0.89 \mu \mathrm{g} / \mathrm{ml}$ degree of toxicity at 24,48 , and $72 \mathrm{~h}$, respectively (Table 6). Lethal dose of C. plurinodis at $50\left(\mathrm{LD}_{50}\right)$ showed $81.66 \pm 1.40$, $135.09 \pm 1.30$, and $120.02 \pm 0.90 \mu \mathrm{g} / \mathrm{ml}$ degree of toxicity at 24,48 , and $72 \mathrm{~h}$, respectively (Table 6 ).

We also observed that there is no information in the literature on the microbial and cytotoxic effect of both plants leaves extracts. The use of cell-based screening assays has proven more relevant in predicting response of organisms to drug effect [39]. More also, evaluating cellular toxicity on the eukaryotic cell culture has been recognized as the model system of choice to get an approximation of toxicity [40]. The $\mathrm{LD}_{50}$ (lethal dose, $50 \%$ ) indicates the quantity of extracts/compounds that, if administered to a population of organisms, will cause $50 \%$ of the organisms to perish. A high $\mathrm{LD}_{50}$ implies it would take a large quantity of the extract to cause a toxic response, while small $\mathrm{LD}_{50}$ values are highly toxic and could be dangerous. It was observed that the dose of both plants extracts appeared to be more toxic after $24 \mathrm{~h}$.

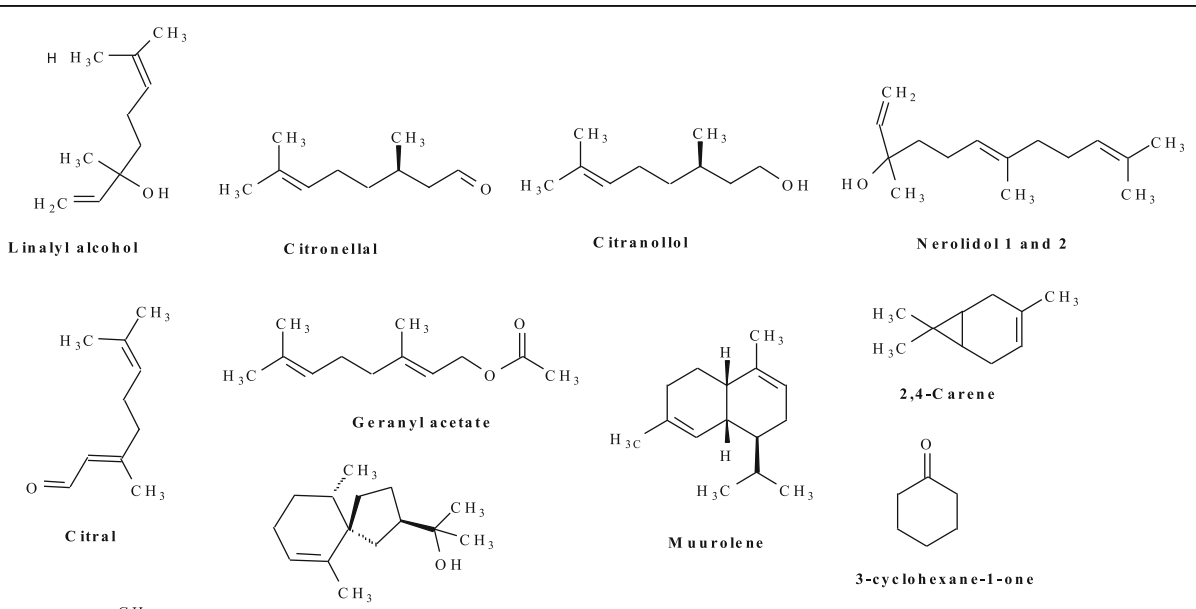

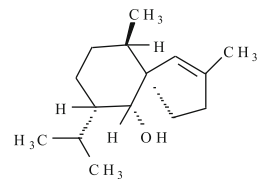
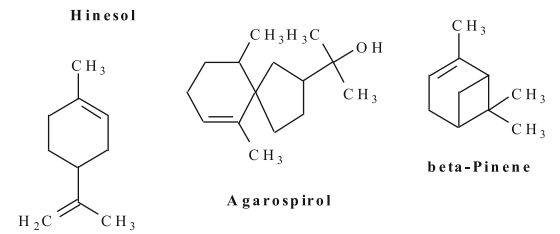

beta-Pinene Agarospirol
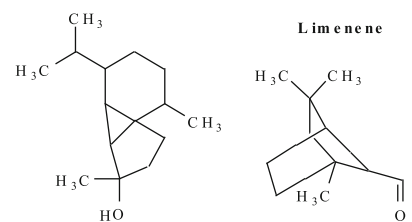

4-epi-cubedol

hotocitral B<smiles>C=CC(=C)CCC=C(C)C</smiles><smiles>Oc1cccc2ccccc12</smiles>

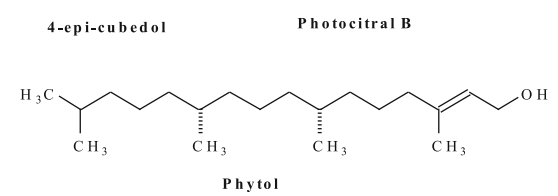

Phytol

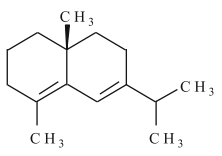<smiles>C=CC(C=C(C)C)C(=C)C</smiles>

Santolina triene

Fig. 2 Some of the identified phyto-compounds that possesses antibacterial activity 
Cymbopogon validis at $24 \quad \mathrm{~h} \quad(\log 0.85 \quad \pm \quad 0.02$; $\left.\mathrm{LD}_{50}=74.87 \pm 1.41\right)$, was found more toxic than the treatment at $48 \mathrm{~h}\left(\log 1.88 \pm 0.01 ; \mathrm{LD}_{50}=139.07 \pm 1.29\right)$ and $72 \mathrm{~h}\left(\log 2.09 \pm 0.00 ; \mathrm{LD}_{50}=122.06 \pm 0.89\right)$. Cymbopogon plurinodis at $24 \mathrm{~h}(\log 0.18 \pm 0.02$; $\mathrm{LD}_{50}=81.66 \pm 1.40$ ) was also found more toxic than the treatment at $48 \mathrm{~h}\left(\log 1.69 \pm 0.01 ; \mathrm{LD}_{50}=135.09 \pm 1.30\right)$ and $72 \mathrm{~h}\left(\log 2.11 \pm 0.00 ; \mathrm{LD}_{50}=120.02 \pm 0.90\right)$. Cell-based lethality assay is an indication of cytotoxicity, bactericidal and various pharmacologic actions. The $\mathrm{LD}_{50}$ values obtained in the current study indicates that the plants extracts have high pharmacological activities [41, 40].

The activities observed against bacterial strains and the human Chang liver cell may be ascribed to the phytocompounds identified in both plants extracts. For example, inhaling linalool has been said to reduce stress-elevated level of neutrophils and lymphocytes in laboratory rats [42]. Citronellal, Citral, Geraniol, Geranyl acetate, Muurolene, Gleenol, Hinesol, Agarospirol, 4-epi-cubedol, Carveol, Photocitral B, Cubedol, Phytol, Epiglobulol, 2,4-Carene and Nerolidol are analgestic that can help reduce pain from strenuous activities and athletics, as well as toothaches, headaches, cough, cold, influenza, fever, and various poxes and inflammation, which can lead to many chronic diseases [27, 43, 44, 28] Fig. 2.

\section{Conclusion}

The extracts of C. validis and C. plurinodis exhibited invitro antibacterial (both Gram-negative and positive species) activity. The major phyto-compounds revealed by GC-MS analysis are believed to be responsible for the antibacterial activity. However, since both plants extracts were toxic to the human Chang liver cells, we recommend that these plants extracts should be used with caution, and further studies using in-vivo (animal model) approach should be conducted to confirm this finding.

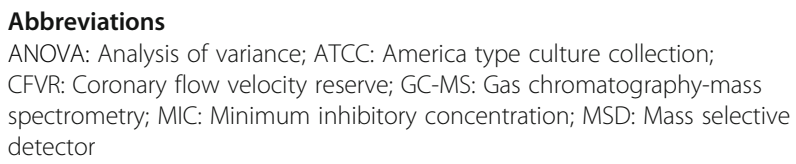

\section{Acknowledgements}

The authors are grateful to Professor Maryna van de Venter from Nelson Metropolitan University for providing the human Chang liver cell lines.

\section{Funding}

This work was supported by the National Research Foundation in South Africa (2014SA02331).

\section{Availability of data and materials}

Data and material are included in the article.

\section{Authors' contributions}

BEO conceived, accomplished, designed the study, responsible for the collection of plant materials, carried out $90 \%$ of the experiments, and drafted the manuscript. MV supervised the entire study, assisted with the study concept and made substantial contribution to the revision of the manuscript. Both authors read and approved the final manuscript.

\section{Competing interests}

The authors declare that they have no competing interests.

\section{Consent for publication}

Not applicable.

\section{Ethics approval and consent to participate}

On behalf of the University of Fort Hare's Research Ethics Committee (UREC), I Professor Gideon de Wet, Dean of Research, hereby give ethical approval in respect of the undertakings contained in the above mentioned project and research instruments. Should any other instruments be used, these require separate authorization. The researcher may therefore commence with the research as from the 25 February, 2014. The certificate reference number is BRAOS1OMUO1.

\section{Publisher's Note}

Springer Nature remains neutral with regard to jurisdictional claims in published maps and institutional affiliations.

\section{Author details}

${ }^{1}$ Department of Biochemistry and Microbiology, University of Fort Hare, Private Bag X1314, Alice 5700, South Africa. ${ }^{2}$ Department of Livestock and Pasture Science, University of Fort Hare, Private Bag X1314, Alice 5700, South Africa.

Received: 15 June 2016 Accepted: 15 March 2017

Published online: 02 June 2017

\section{References}

1. Avoseh O, Oyedeji O, Rungqy P, Nkeh-Chungag B, Oyedeji A. Cymbopogon species; ethnopharmacology, phytochemistry and the pharmacological importance. Int J Mol Sci. 2015;20:7438-53.

2. Kumar A, Kumar A, Thakur P. Antibacterial activity of green tea (Camellia sinecsis) extracts against various bacteria isolated from environmental sources. Recent Res Sci and Technol. 2012;4:19-23.

3. Okoh OO, Sadimenko AP, Afolayan AJ. Comparative evaluation of the antibacterial activities of the essential oils of Rosemarinus officinalis $L$. obtained by hydro-distillation and solvent free microwave extraction methods. Food Chem. 2010;120:308-12.

4. Oyedemi SO, Afolayan AJ. Antibacterial and antioxidant activities of hydroalcoholic stem bark extract of Schotia latifolia Jacq. Asian Pakistani J Med. 2011;4(2):952-8.

5. Lawal O, Grierson DS, Afolayan AJ. Phytotherapeutic information of plants used for the treatment of tuberculosis in Eastern Cape Province, South Africa. Evidence-Based Com and Alt Med. 2014;2014:11. doi:10.1155/2014/ 735423.Article ID 735423

6. Sen A, Batra A. Evaluation of antimicrobial activity of different solvent extracts of medicinal plant: Melia azedarach L. Int J Curr Pharm Res. 2012; 4(2):67-73.

7. Buwa LV, van Staden J. Antibacterial and antifungal activity of traditional medicinal plants used against venereal diseases. J Ethanopharmacol. 2012; 103:139-42.

8. Bakkali F, Averbeck S, Averbeck D, Idaomar M. Biological effects of essential oils - a review. Food and Chem Toxicol. 2008;46(2):446-75.

9. Shah G, Shri R, Panchai V, Sharma N, Singh B, Mann AS. Scientific basis for the therapeutic use of Cymbopogon citratus, Stapf (lemongrass). Int J Adv Technol and Eng Res. 2011;2(1):3-8

10. Torregorossa A, Dearing MD. Caching as a behavioural mechanism to reduce toxin intake. J Mammal. 2009;90(4):803-10.

11. Giweli A, Dzamic AM, Sokovic M, Ristic MS, Marin PD. Antimicrobial and antioxidant activities of essential oils of Satureja thymbra growing wild in Libya. Mol. 2012;17:4836-50.

12. Bicchi C, Rubiolo P, Ballero M, Sanna C, Matteodo M, Esposito F, Zinzula L, Tramontano E. HIV-1 inhibiting activity of the essential oil Ridolfia segetum and Oenanthe crocata plant medicine. J Chem and Mat Res. 2009;75:1331-5.

13. El-sharkawy ER. Anticancer effect and seasonal variation in oil constituents of Santolina chamaecyparissu. Chem and Mat Res. 2014;6(4):2224-3224.

14. Miller JA, Thompson PA, Hakim IA, Chow HHH, Thomson CA. D- limonene: a bioactive food component from citrus and evidence for a potential role in breast cancer prevention and treatment. Oncol Rev. 2011;5(1):31-42. 
15. Bhuiyan MNI, Akter F, Chowshury JU, Begum J. Chemical constituents of essential oils from aerial parts of Adenosma capitatum and Limnophila aromatic. Bangladesh J Pharmacol. 2010;5:13-6.

16. Nakamura A, Fujiwara S, Matsumoto I, Abe K. Stress repression in restrained rats by (R)-(-)-linalool inhalation and gene expression profiling of their whole blood cells. J Agric Food Chem. 2009;57(12):5480-5.

17. Huo M, Cui X, Xue J, Chi G, Gao R, Deng X, Guan S, Wei J, Soromou L. Antiinflammatory effects of linalool in RAW 264.7 macrophages and lipopolysaccharides induced lung injury model. J Surg Res. 2013;180(1):47-54

18. Ahmad F, Khan MA, Zafar MAM, Khan A. Taxonomic utilization of anatomical characters in tribe Andropogoneae (Poacease) based on transverse sections of leaves. J Med plants Res. 2010;4(14):1349-58.

19. Ci X, Chu X, Wei M, Yang X, Cai Q, Deng X. Different effects of farrerol on an OVA-induced allergic asthma and LPSinduced acute lung injury. PLoS One. 2012;7(4):e34634.

20. Palombo EA. Traditional medicinal plant extracts and natural products with activity against oral bacteria: potential application in the prevention and treatment of oral diseases. Evidence-Based Com and Alt Med. 2011;680354:1-15.

21. Astani A, Reichling J, Schnitzler P: Screening for antiviral activities of isolated compounds from essential oils. Evidence-Based Com and Alt Med. 2011, Article ID 253643, doi: 10.253643/1093.

22. Hamdan DI, Abdulla RH, Mohamme ME, El-Shazly AM. Chemical composition and biological activity of essential oils of Cleopatra mandarin (citrus reshni) cultivated in Egypt. J Pharmacog and Phyto. 2013;5(5):83-90.

23. Bueno J, Escobar P, Martinez JR, Leal SM, Stashenko EE. Composition of three essential oils, and their mammalian cell toxicity and antimycobacterial activity against drug resistant-tuberculosis and nontuberculous mycobacteria strains. Nat prod Com. 2011;6(11):1743-8.

24. Silva-Brum LF, Emanuelli T, Souza DO, Elisabetsky E. Effect of linalool on glutamate release and uptake in mouse cortical synaptosomes. Neurochem Res. 2001;26:191-4.

25. Arruda DC, d'Alexandri FL, Katzin AM, Uliana SRB. Antileishmanial activity of the terpene Nerolidol. Antimicrobial Agent and Chemo. 2005;49(5):1679-87.

26. Omoruyi BE, Afolayan AJ, Bradley G. Chemical composition profiling and antifungal activity of the essential oil and plant extracts of Mesembryanthemum edule (L.) bolus leaves. Afri J Trad and Com Alt Med. 2014;11(4):19-30.

27. Gloerich J, van den Brink DM, Ruiter JPN, van Vlies N, Vaz FM. Metabolism of phytol to phytanic acid in the mouse, and the role of PPARa in its regulation. J Lipid Res. 2007;48:77-85.

28. Santos CCDP, Salvadori MS, Mota VG, Costa LM, de Almeida AAC, de Oliveira GAL, Costa JP, de Sousa DP, de Freitas RM, de Almeida RN. Antinociceptive and antioxidant activities of phytol in vivo and in vitro models. Neuroscience J. 2013; http://dx.doi.org/10.1155/2013/949452.

29. Bentley FK, Garci'a-Ce'n JG, Chen HC. Paradigm of monoterpene (bphellandrene) hydrocarbons production via photosynthesis in cyanobacteria. BioEner Res. 2013;6:917-29.

30. Deba F, Xuan TD, Yasuda M, Tawatu S. Chemical composition and antioxidant, antibacterial and antifungal activities of the essential oils from Bidens pilosa. Linn Var Radiata Food contrl. 2008;19:346-52.

31. Imelouane B, Amhamdi B, Wathelet JP, Ankit M, Khedid K, Bachiri El A. Chemical composition and antimicrobial activity of essential oil of thyme (Thymus vulgaris) from Eastern Morocco. Int J Agr Biol. 2009;11:205-8.

32. Nezhadali A, Nabavi M, Rajabian M, Akbarpour M, Pourali P, Amini F. Chemical variation of leaf essential oil at different stages of plant growth and in vitro antibacterial activity of Thymus vulgaris Lamiaceae, from Iran. Beni-Suef University J Basic Appl Sci. 2014;3(2):87-92.

33. Hajji M, Jarraya R, Lassoued I, Masmoudi O, Damak M, Nasri M. GC/MS and LC/ MS analysis, and antioxidant and antimicrobial activities of various solvent extracts from Mirabilis jalapa tubers. Process Biochem. 2010;45:1486-93.

34. Oussalah M, Caillet S, Saucier L, Lacroix M. Antimicrobial effects of selected plant essential oils on the growth of a Pseudomonas putida strain isolated from meat. Meat Sci. 2006;73:236-44.

35. Oussalah M, Caillet S, Saucier L, Lacroix M. Inhibitory effects of selected plant essential oils on the growth of four pathogenic bacteria, E. coli 0157:H7, S. typhimurium, S. aureus and L. monocytogenes. Food Control. 2006;18(5):414-20.

36. Hammer KA, Carson CF, Riley TV. Antimicrobial activity of essential oils and other plant extracts. J Appl Microbiol. 1999;86:985-90.

37. Wei L, Wee W. Chemical composition and antimicrobial activity of Cymbopogon nardus Citronella essential oil against systemic bacteria of aquatic animals. Iran J Microbiol. 2013;5(2):147-52.
38. Paliwal A, Gujar RK, Sharma HN. Analysis of liver enzymes in albino rats under stress of I-Cyhalothrin and Nuvan toxicity. Biol and Med. 2009;1:70-3.

39. El-Sharkawy ER, Matloub AA, Atta EM. Cytotoxicity of new flavonoid compound isolated from Farsetia aegyptia. Int J Pharm Sci Inv. 2013;2:23-7.

40. Mthethwa NS, Oyedeji BAO, Obi CL, Aiyegoro OA. Anti-staphylococcal, antiHIV and cytotoxicity studies of four South African medicinal plants and isolation of bioactive compounds from Cassine Transvaalensis (Burtt. Davy) codd. BMC Com and Alt Med. 2014;14:512. http://www.biomedcentral.com/ $1472-6882 / 14 / 512$

41. Nasri H, Shirzad H. Toxicity and safety of medicinal plants. J HerMed Pharmacol. 2013;2:21-2

42. Nakamura A, Fujiwara S, Matsumoto I, Abe K. Stress repression in restrained rats by (R)-(-)- linalool inhalation and gene expression profiling of their whole blood cells. J Agri and Food Chem. 2009;57(19):5480-5.

43. Orhan IE, Ozcelik B, Kartal M, Kan Y. Antimicrobial and antiviral effects of essential oils from selected Umbelliferae and Labiatae plants and individual essential oil components. Turk J Biol. 2012;36:239-46.

44. Singh D, Kumar TRS, Gupta VK, Chaturvedi P. Antimicrobial activity of some promising plant oils, molecules and formulations. Indian J Exp Biol. 2012:55:714-7.

\section{Submit your next manuscript to BioMed Central and we will help you at every step:}

- We accept pre-submission inquiries

- Our selector tool helps you to find the most relevant journal

- We provide round the clock customer support

- Convenient online submission

- Thorough peer review

- Inclusion in PubMed and all major indexing services

- Maximum visibility for your research

Submit your manuscript at www.biomedcentral.com/submit
C Biomed Central 